\title{
Measuring S100 protein and neurone specific enolase in melanocytic tumours using video image analysis
}

\author{
R A WILliAMS,* J RODE, $\dagger$ A P DHILlON, $\dagger$ L R JARVIS, J M SKINNER, \\ O JAMAL
}

From the *Wangaratta District Base Hospital, Wangaratta, Victoria, Australia, The $†$ Bland-Sutton Institute of Pathology, Middlesex Hospital, London, and the Department of Pathology, Flinders Medical Centre, Bedford Park, Australia

SUMMARY Using a computed video image analysis system, the staining intensity for both neurone specific enolase (NSE) and S100 protein was measured in sections from 19 malignant melanomas and 16 benign melanocytic lesions. The results of this study confirm previous reports that NSE and S100 protein are useful markers for malignant melanoma. NSE staining intensity in the cases of malignant melanoma was significantly higher than that in benign naevi $(p=0.011)$. Intensity of staining for S100 protein was not significantly higher in the malignant melanomas. There was, however, a significant S100 gradient when comparing superficial and deep intradermal portions of these tumours $(p=0.003)$. This feature was not seen in benign naevi. The greatest intensity of $S 100$ protein staining was found in the deeper portions of the malignant melanomas. This gradient difference was not seen with staining for NSE. Although it seems that the overall intensity of staining for NSE is more effective in differentiating between benign and malignant lesions, the difference in staining intensity between the superficial and deep portions of the tumour may be the better indicator of adverse behaviour in lesions in which the diagnosis of malignancy is uncertain

Behaviour in individual cases of malignant melanoma is exceedingly difficult to predict, although the depth of invasion seems to be one of the most reliable prognostic indicators at present. ${ }^{1-4}$ In a previous study we reported that staining intensity and pattern for neurone specific enolase (NSE) were of diagnostic value in differentiating benign from malignant melanocytic lesions and that differences in staining intensity were of potential prognostic value. ${ }^{5}$

There are problems with this approach. Not least is the fact that the studies, which have so far been undertaken, entail subjective assessment of the level of staining intensity and may therefore not be reproducible between laboratories or even between observers in the same laboratory.

This can be overcome by having an objective measure of staining intensity rather than the usual subjective plus scale scoring. In this study the optical density of staining in tumour tissue was measured using a microcomputer video image analysis system. ${ }^{6}$ Nineteen cases of malignant melanoma and 16 benign melanocytic lesions were stained for both NSE and S100 protein and the average staining intensity between the superficial, deep, and total tumour regions was examined.

Accepted for publication 30 April 1986

\section{Material and methods}

Archived material from the files of the Flinders Medical Centre was used for this study. The tumours selected came from a batch reviewed by a panel of four consultant pathologists. Only tumours in which there was unanimous independent agreement as to the histological diagnosis were measured.

Sections of 19 malignant melanomas and 16 benign compound naevi that had been formalin fixed and paraffin embedded were cut at $5 \mu \mathrm{m}$ and stained to show the presence of NSE and S100 protein.

STAINING

The details of the method have been described previously. ${ }^{7}$ Rabbit antihuman NSE was used at a dilution of $1 / 100$ for 30 minutes and rabbit antibovine S100 (which cross reacts with human S100) at $1 / 200$ for 120 minutes. Alkaline phosphatase conjugated goat antirabbit antibody was used at a dilution of 1/50 for 30 minutes and immunolocalisation developed with napthol AS-MX phosphoric acid with fast red TR salt (Sigma). The red reaction product avoids confusion in interpretation of staining in those tumours producing melanin. The entire set of slides for this experiment were stained as one batch for both NSE and S100 protein so that intensity of staining 


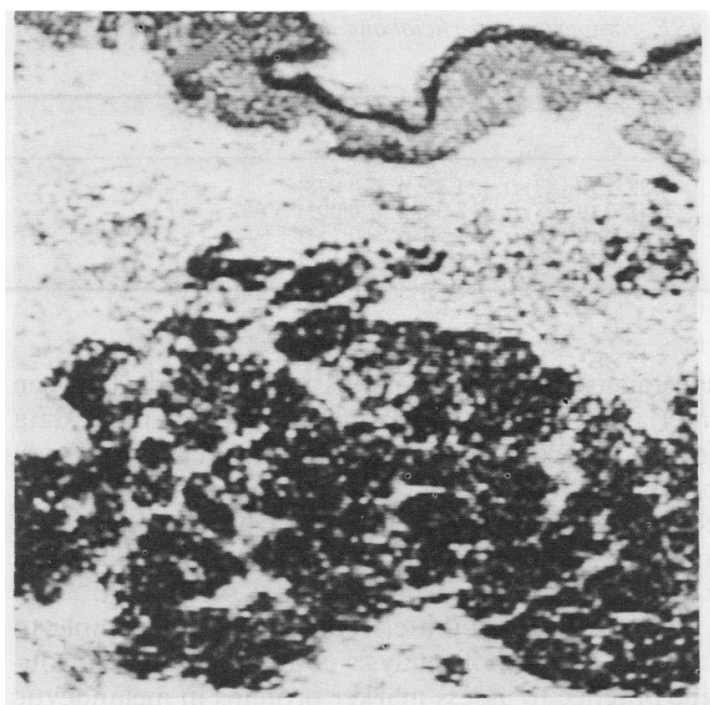

Fig 1 Digital grey level image of sectioned tumour stained for $S 100$ protein. Complete depth of tumour was contained within field.

could be compared without the problem of differences due to interbatch technical variations.

\section{ANALYSIS}

The image analysis was performed using a system designed in our laboratory comprising a solid state

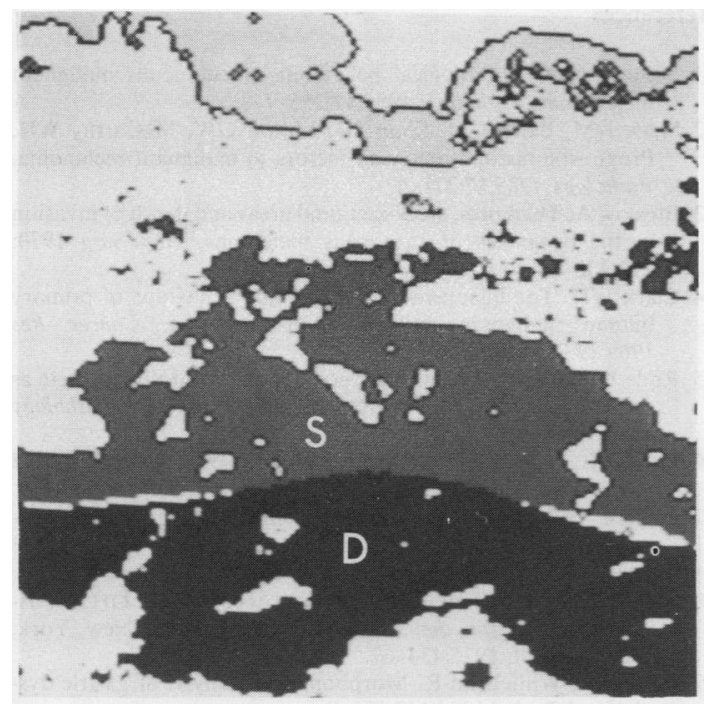

Fig 2 Segmentation of staining regions by grey value to give binary image followed by delineation of superficial $(S)$ and deep (D) regions at about $50 \%$ of tumour depth by interactive editing to allow computer to distinguish independent masks over grey level image for measurement of area and integrated optical density. camera, video digitiser, microcomputer, and image analysis software designed specifically for densitometric measurement. ${ }^{6}$ The software permitted the integrated optical density and area of images to be measured in standardised arbitrary units. To control densitometric errors calibration procedures were used to ensure that the pixel values were linear in proportion to the illumination intensity. Correction for uneven illumination was performed by storing an image of the "white" background before measurement. The pixel values from the store were retrieved and used to correct every subsequent scan. Measurements were made using a $550 \mathrm{~nm}$ narrow band (10 $\mathrm{nm}$ band width) interference filter and a $\times 2.5$ objective lens. The whole or major depth of each tumour was contained within the field of view. Up to four such fields were selected for each case and scanned to give digital images with 64 levels of grey for densitometric analysis (fig 1). The regions of staining were automatically segmented by the computer, based on the relative density of the stain above that of the unstained tissue, to give a binary image. To permit quantitation of the gradient of staining intensity the sectioned tumours were arbitrarily divided into superficial and deep regions, using the thickest portions of the tumour, by a line running parallel to the surface of the skin at a level of $50 \%$ of the tumour depth. All the tumour above this line was designated as "superficial" tumour mass and that below the line as "deep" tumour mass. Superficial and deep portions of the tumour and melanocytic cells from both regions were delineated by interactive editing of the binary image to give independent masks that defined the regions of the grey level image to be measured by the computer (fig 2). The staining intensity, expressed as average optical density, was calculated from the area and integrated optical density measurements. The time required for analysis was about 10 minutes per case.

Statistical analysis was performed with a two tailed Student's $t$ test for unpaired groups, using the programs outlined in Statistical Package for the Social Sciences. ${ }^{8}$

\section{Results}

S100 PROTEIN STAIN

For the whole sectioned area of tumour the mean staining intensity for the malignant lesions was not

Table 1 Means (SD) of optical density of immunochemical staining for S100 protein and NSE, comparing groups of benign and malignant lesions

\begin{tabular}{lll}
\hline & Benign $(n=16)$ & Malignant $(n=19)$ \\
\hline S100 & $0.3746(0 \cdot 117)$ & $0.3939(0.109)(t=0.53 ; \mathrm{p}=0.596)$ \\
NSE & $0.3042(0 \cdot 100)$ & $0.3959(0.100)(t=2 \cdot 70 ; \mathrm{p}=0.011)^{*}$ \\
\hline
\end{tabular}

*Significance at $95 \%$ confidence level. 
Table 2 Mean optical densities of staining for S100 protein and NSE, comparing superficial and deep regions for both benign and malignant lesions

\begin{tabular}{lll}
\hline & Superficial & Deep \\
\hline S100 benign $(\mathrm{n}=16)$ & $0.3653(0.115)$ & $0 \cdot 3839(0 \cdot 123)(t=1 \cdot 75 ; \mathrm{p}=0.098)$ \\
S100 malignant $(\mathrm{n}=19)$ & $0.3752(0.099)$ & $0 \cdot 4162(0 \cdot 123)(t=3 \cdot 36 ; \mathrm{p}=0.003)^{*}$ \\
NSE benign $(\mathrm{n}=16)$ & $0.2970(0.097)$ & $0 \cdot 3144(0 \cdot 106)(t=1 \cdot 50 ; \mathrm{p}=0.154)$ \\
NSE malignant $(\mathrm{n}=19)$ & $0.3888(0.023)$ & $0.4029(0.024)(t=1 \cdot 53 ; \mathrm{p}=0.145)$ \\
\hline
\end{tabular}

*Significant at $95 \%$ confidence level.

significantly different from that of the benign lesions (table 1). For the superficial and deep regions of the tumours the staining intensity was not significantly different in the benign lesions, but for the malignant lesions the staining intensity in the deep region was significantly higher than that in the superficial region (table 2).

\section{NSE STAIN}

For the whole sectioned tumour area the mean staining intensity was significantly higher for malignant lesions (table 1). For the superficial and deep regions, the staining intensity was not significantly different from that of either the malignant or benign groups (table 2).

\section{Discussion}

The results verify previous subjective observations that the intensity of staining for NSE differs significantly between benign and malignant lesions. Similarly, for the malignant lesions there was an expected increase in staining intensity for $\mathrm{S} 100$ protein but this was only slight and was not significant (table 1).

The results suggest that in practice measurement of the intensity of neurone specific enolase staining may be more useful than that of $\mathrm{S} 100$ protein for discerning between benign and malignant lesions. Staining for S100 protein, however, enables the S100 gradient (superficial to deep) to identify malignant tumours, with the higher staining intensities in the deeper layers identifying the invasive component. The ratio of $\mathrm{S} 100$ protein staining densities between superficial and deep regions may therefore be a more convenient indicator of behaviour in any one case. The study showed that staining intensities for NSE and S100 protein in melanocytic tumours can be measured by a simple, quick, and accurate image analysis technique using a microcomputer system, as described. The measurement of staining intensity by image analysis eliminates the error caused by subjectivity, when using arbitrary scales to assess visual impressions of staining intensity.

It follows that if such measurements are made in a standardised way with appropriate batch control specimens valid comparisons can be made between interlaboratory data. This means that reference data from a large number of cases of known diagnosis can be compiled, including response to treatment and outcome. Equations can then be made using discriminant analysis, which provide objective predictions for any case, if the same measurements are performed. ${ }^{9}$

As it is fast, accurate, economical, and simple to use, this method of analysis could be applied in routine practice to assess marker proteins in melanocytic and other tumours.

This work was supported in part by a grant from the Wangaratta Regional Pathology Service Special Purpose Trust Fund, at the Wangaratta District Base Hospital, Victoria, Australia, and by the Cancer Research Campaign, United Kingdom. We thank D尺 RJ Thompson, department of clinical biochemistrip Addenbrooke's Hospital, Cambridge, England, fo 5 the generous supply of antihuman NSE.

\section{References}

1 Sondergaard K. Biological behaviour of cutaneous malignant melanomas. Pathology 1985;17:255-7.

2 Shaw HM, Balch CM, Soon S-J, Milton GW, McCarthy WH Prognostic histopathological factors in malignant melanoma. Pathology 1985;17:271-4.

3 Breslow A. Thickness, cross-sectional areas and depth of invasion in the prognosis of cutaneous melanoma. Ann Surg 1970; 172:902-8.

4 Clark WH. The histogenesis and biologic behaviour of primary human malignant melanoma of the skin. Cancer Res 1969;29:705-26.

5 Rode J, Dhillon AP. Neurone specific enolase and S100 protein as possible prognostic indicators in melanoma. Histopathology 1984;8:1041-52.

6 Jarvis LR. A microcomputer system for video image analysis and diagnostic microdensitometry. Anal Quant Cytol (in press).

7 Dhillon AP, Rode J. Immunohistochemical studies of S100 protein and other neural characteristics expressed by granular cell tumour. Diagnostic Histopathology 1983;6:23-8.

8 Nie NH, Hull CH, Jenkins JG, Steinbrenner K, Bent DH. Statistical package for the social sciences. 2nd ed. New York: McGraw-Hill, 1975:434-67.

9 Jarvis LR, Whitehead R. Morphometric analysis of gastric dysplasia. J Pathol 1985;147:133-8.

Requests for reprints to: Dr J Rode, Department of Histopathology, The Bland-Sutton Institute of Pathology, The Middlesex Hospital Medical School, London W1P 7PN, England. 